\title{
Management Strategies for POSEIDON Group 2
}

\author{
Sesh Kamal Sunkara ${ }^{1 *}$, G. A. Ramaraju² and Mohan Shashikant Kamath ${ }^{3}$ \\ 'Division of Women's Health, Faculty of Life Sciences and Medicine, King's College London, London, United Kingdom, \\ ${ }^{2}$ Center for Assisted Reproduction, Krishna IVF Clinic, Visakhapatnam, India, ${ }^{3}$ Department of Reproductive Medicine, \\ Christian Medical College, Vellore, India
}

OPEN ACCESS

Edited by:

Sandro C. Esteves,

Androfert, Andrology and Human

Reproduction Clinic, Brazil

Reviewed by:

Alberto Vaiarelli,

GENERA reparto di PMA della Clinica

Valle Giullia, Italy

Giuliano Marchetti Bedoschi,

University of São Paulo, Brazil

${ }^{*}$ Correspondence:

Sesh Kamal Sunkara sesh.sunkara1@nhs.net; sesh.sunkara@kcl.ac.uk

Specialty section:

This article was submitted to Reproduction,

a section of the journal

Frontiers in Endocrinology

Received: 12 December 2019

Accepted: 18 February 2020

Published: 27 February 2020

Citation:

Sunkara SK, Ramaraju GA and Kamath MS (2020) Management Strategies for POSEIDON Group 2.

Front. Endocrinol. 11:105.

doi: 10.3389/fendo.2020.00105
Although individualization of ovarian stimulation aims at maximal efficacy and safety in assisted reproductive treatments, in its current form it is far from ideal in achieving the desired success in women with a low prognosis. This could be due a failure to identify such women who are likely to have a low prognosis with currently used prognostic characteristics. Introduction of the patient-oriented strategies encompassing individualized oocyte number (POSEIDON) concept reinforces recognizing such low prognosis groups and stratifying in accordance with important prognostic factors. The POSEIDON concept provides a practical approach to the management of these women and is a useful tool for both counseling and clinical management. In this commentary, we focus on likely management strategies for POSEIDON group 2 criteria.

Keywords: POSEIDON classification, POSEIDON group 2, poor responder, suboptimal responder, IVF (ICSI)

\section{INTRODUCTION}

Success following assisted reproductive treatments (ART) has improved significantly since the early years of in vitro fertilization (IVF) treatment. The notable contribution to this success is the introduction of ovarian stimulation into ART in the early 1980s $(1,2)$. Soon after the introduction of ovarian stimulation, it became apparent that women varied in their response to stimulation. To this end a low responder was first described as being associated with low serum oestradiol levels and requiring higher gonadotrophin stimulation doses (3). Since then, there had been varied descriptions and terminologies such as poor ovarian response (POR), low response, inadequate response, suboptimal response with numerous definitions, several criteria and different thresholds.

A review in 2000 enlisted around 28 criteria used for the definition of POR (4) and a latter review nearly 10 years later reinforced the issue of lacking uniform criteria for defining POR with 41 definitions being used in 47 RCTs that had since been published on the topic (5). Discrepancies in the definition lead to clinical heterogeneity among studies on POR leading to inconsistent and inconclusive findings (6). This lead to researchers and clinicians calling for a unified definition of POR leading to the publication of the ESHRE consensus, Bologna criteria definition of POR (7).

However, there has been skepticism whether the ESHRE consensus, the Bologna criteria for defining POR is fit for purpose and whether the new consensus definition mitigated clinical heterogeneity. The ESHRE consensus definition of POR considers proven poor responders based on previous cycle performances and predicted poor responders as one category, does not consider suboptimal response, does not factor in female age and the oocyte competence in terms of embryos aneuploidy rate (8). Additionally, it comprises of several subpopulations with varied baseline characteristics (9). Furthermore, the Bologna criteria encompasses a very poor prognosis group that is associated with very low live birth rates $(10,11)$ raising the interrogation if any interventions could enhance clinical outcomes for these women with very poor prognosis $(12,13)$. 


\section{CONCEPT OF INDIVIDUALIZED OVARIAN STIMULATION}

The main objective of individualization of ovarian stimulation (OS) is to offer women the best treatment tailored to her own unique characteristics, thus maximizing the chances of pregnancy and eliminating the iatrogenic and avoidable risks resulting from ovarian stimulation (14). It currently entails categorizing women based on their predicted response in order to individualize OS regimens. Women can be identified as having an expected poor response, normal response or a high response based on ovarian reserve tests (ORTs). Among the various ORTs including basal FSH, basal oestradiol, inhibin $\mathrm{B}$, antral follicle count (AFC), and anti-mullerian hormone (AMH), AFC, and $\mathrm{AMH}$ have the highest accuracy for the prediction of either a poor or a high response following ovarian stimulation (1517). However, whether the categorization into the three broad categories of poor, normal and high response is sufficient to categorize all women in an ART programme has been questioned with evidence-based suggestions to refine the categorization by recognizing the suboptimal responder (18). Suboptimal response is the group between poor response with $\leq 3$ oocytes and normal response with 10-15 oocytes. These women with 49 oocytes have a better prognosis over poor responders but have a lower prognosis compared to normal responders. Given that poor responders have a very low prognosis with most interventions being futile, it would be justifiable to focus research and interventions toward other low prognosis groups such as the suboptimal responder. This further lead to the notion of "patient oriented strategies encompassing individualized oocyte number"-POSEIDON concept. The recent publication in Frontiers in Endocrinilogy by Conforti and colleagues hughlights the need for intervention studies to test the POSEIDON concept, particularly for groups 1 and 2 where benefit is more likely in the context of a good ovarian reserve (19). This paper discusses interventions in the context of POSEIDON group 2 women that would merit further research.

\section{POSEIDON CONCEPT: THE WHY, THE WHAT AND THE HOW}

A systematic review and meta-analysis on predictive factors in IVF evaluated nine common predictors and found the following factors of female age, duration of infertility, basal follicle stimulating hormone (FSH) levels, the number of retrieved oocytes, and embryo quality to be associated with the chances of pregnancy (20). Older female age, longer duration of infertility, higher basal FSH levels were negative predictors whereas higher number of oocytes and good embryo quality were positive predictors. There has been consistent evidence of a strong association between number of oocytes retrieved and live birth reinforcing that the number of oocytes is an important prognostic variable for IVF success (21-24). It is therefore paramount that the OS regimens optimize number of oocytes retrieved to maximize success.
Younger women have a favorable prognosis in achieving a live birth compared to older women. An important reason for this is the increase in aneuploid embryos and consequent decrease in euploid embryos with increasing female age. Whereas, female age influences the embryo euploidy rate, euploidy rate remains stable in relation to the embryo cohort sizes, thereby resulting in more euploid embryos with higher number of embryos (25). It therefore becomes apparent that female age, ovarian reserve, ovarian response to stimulation and number of oocytes retrieved are overriding factors determining the success of ART. Ovarian stimulation regimen should therefore aim to enhance ovarian response to stimulation, particularly for the low prognosis group of patients extending to the suboptimal responder.

The POSEIDON stratification is aimed at clinical management by considering the most important prognostic factors and stratifying women accordingly. Group 1: women aged $<35$ years with adequate ovarian reserve $(\mathrm{AFC} \geq 5, \mathrm{AMH}$ $\geq 1.2 \mathrm{ng} / \mathrm{ml}$ ) with an unexpected poor response ( $<4$ oocytes) or a suboptimal response (4-9 oocytes); Group 2: women aged $\geq 35$ years with adequate ovarian reserve $(\mathrm{AFC} \geq 5, \mathrm{AMH} \geq$ $1.2 \mathrm{ng} / \mathrm{ml}$ ) with an unexpected poor response ( $<4$ oocytes) or a suboptimal response (4-9 oocytes); Group 3: women aged $<$ 35 years with poor ovarian reserve $(\mathrm{AFC}<5, \mathrm{AMH}<1.2 \mathrm{ng} /$ $\mathrm{ml}$ ); Group 4 : women aged $\geq 35$ years with poor ovarian reserve $(\mathrm{AFC}<5, \mathrm{AMH}<1.2 \mathrm{ng} / \mathrm{ml})(26)$.

\section{MANGAMENT OF POSEIDON GROUP 2 WOMEN}

The aim of defining the POSEIDON groups is to individualize therapeutic approaches by fine tuning OS in terms of the right pituitary suppression regimen, the ideal gonadotrophin selection, along with dosage and optimize ovarian response and number of oocytes to obtain a euploid embryo with the highest implantation potential for transfer. POSEIDON classification reinforces the avoidance of iatrogenic suboptimal response underpinning likely genetic variants such as FSH receptor polymorphism (27), variant luteinising hormone- $\beta$ (V LH- $\beta$ ) (28) that might benefit from gonadotrophins with different pharmacokinetic profiles and yielding a higher number and competent oocytes for a given dosage $(29,30)$. The broad stratification based on a female age cutoff, taking cognizance of the declining prognosis in women beyond age of 35 years, is likely to be helpful in clinical decision making for the vast majority of women undergoing IVF below age of 40 years (31).

The long gonadotrophin releasing hormone $(\mathrm{GnRH})$ agonist regimen is associated with a significantly higher oocyte yield over the short GnRH agonist regimen, and as such should be the preferred downregulation regimen with the use of $\mathrm{GnRH}$ agonists (32). Given the concurring evidence of comparable efficacy with the use of the long GnRH agonist and $\mathrm{GnRH}$ antagonist regimens for both general population and poor responder women undergoing IVF (33), either regimens could be recommended for POSEIDON group 2 women by extrapolating current evidence. As the aim is to improve egg numbers, these women may benefit from a higher gonadotrophin dose over the 


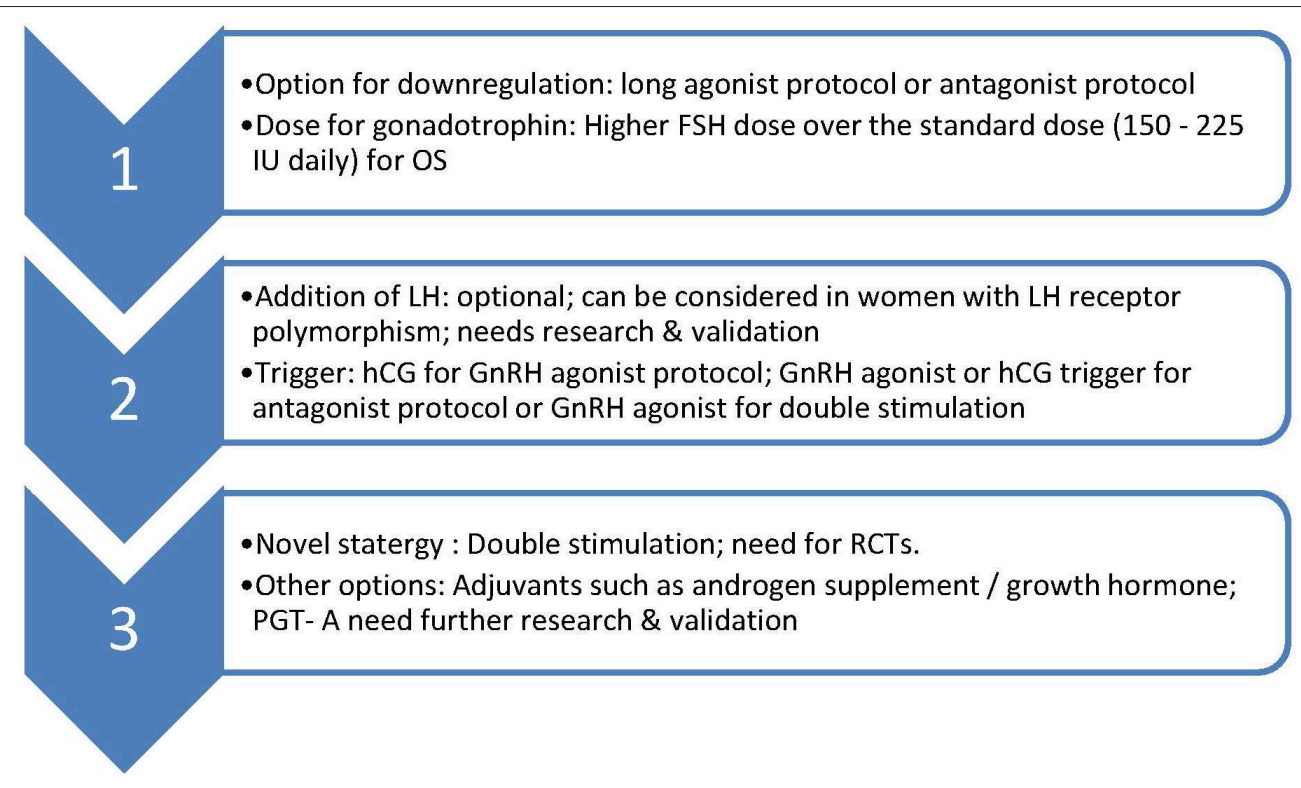

FIGURE 1 | Suggested management of POSEIDON group 2. OS, ovarian stimulation; LH, luteinising hormone; hCG, human chorionic gonadotrophin; GnRH, Gonadotrophin releasing hormone; PGT-A, pre implantation genetic testing-aneuploidy; RCT, randomized controlled trial.

standard $150 \mathrm{IU}-225 \mathrm{IU}$ daily for OS (34). There is the discussion that women in this group have a specific genotype profile accounting for the variability in ovarian response to stimulation that is unexpected based on routine ovarian reserve testing. There has been evidence on the relevance of genetic variants of gonadotropins and their receptors in ovarian stimulation and benefits of increasing FSH dose or adding recombinant $\mathrm{LH}$ for women with a hypo response to recombinant FSH (35) depending upon presence of FSH or LH receptor polymorphisms, respectively (36). Whether FSH or LH receptor polymorphism screening should be offered to all women with adequate ovarian reserve prior to their first IVF treatment depends on prevalence of such polymorphism in this select IVF population and its impact. Further, whether these women are likely to benefit from gonadotrophins with different pharmacokinetic profile such as additional LH activity to FSH needs evaluation with further research into this area.

Dual stimulation is a novel strategy in which double stimulation ("DuoStim") is attempted in the same menstrual cycle (36). Earlier, it was proposed that only one wave of follicular recruitment takes place in an ovarian cycle. It has hence been shown that two and three cohorts of antral follicles are recruited during a menstrual cycle (37-39). Double stimulation has been proposed as one of the treatment statergy for management of POSEIDON group 2 which consists of women $\geq 35$ years with an unexpected POR or suboptimal response. Since aneuploidy rates are higher in this group compared to women $<35$ years, higher oocyte yield is needed to achieve a single euploid embryo. Double stimulation strategies can help in maximizing oocyte yield in a single ovarian cycle. An earlier study has compared the oocyte yield and euploid blastocyst rates following FPS and LPS (40). The study reported no significant difference in retrieved cumulus oocyte complex $(5.1 \pm 3.4$ vs. $5.7 \pm 3.3)$ or euploid blastocyst rates ( 46.9 vs. $44.8 \%$ ). A recent case control included 188 women with poor prognosis who under double stimulation (41). The authors reported fewer oocytes collection (3.6 \pm 2.1 vs. $4.3 \pm 2.8 ; P<0.01)$ and euploid blastocysts $(0.5 \pm 0.8$ vs. $0.7 \pm 1.0 ; P=0.02)$ after FPS compared to LPS. A systematic review which included eight studies and 338 women, reported no compromised in quality or quantity of oocytes retrieved following LPS compared to FPS (42). A "freeze all strategy" is mandatory for double stimulation. It is suggested that double stimulation may reduce the cycle drop out rates in these women with poor or suboptimal response and shorten time to pregnancy (42). Currently, there is very limited data is available on obstetrical and neonatal outcomes following double stimulation.

Double stimulation protocol needs validation in POSEIDON group 2 population along with cost-effectiveness and safety data. Overall, such group of women in POSEIDON group 2 might benefit recognition and whether they could benefit from novel strategies or inteventions such as adjuvant androgen therapies, addition of growth hormone, preimplantation genetic testing for aneuploidy (PGT-A) warrants further research. Management options for POSEIDON group 2 women is summarized in Figure 1.

\section{AUTHOR CONTRIBUTIONS}

SS drafted the manuscript with contribution from GR and MK. All authors approved final version. 


\section{REFERENCES}

1. Trounson AO, Leeton JF, Wood C, Webb J, Wood J. Pregnancies in humans by fertilization in vitro and embryo transfer in the controlled ovulatory cycle. Science. (1981) 212:681-2. doi: 10.1126/science.7221557

2. Jones HW Jr, Jones GS, Andrews MC, Acosta A, Bundren C, Garcia J, et al. The program for in vitro fertilization at Norfolk. Fertil Steril. (1982). 38:14-21. doi: 10.1016/S0015-0282(16)46390-9

3. Garcia J, Acosta A, Andrews MC, Jones GS, Jones HW Jr, Mantzavinos T, et al. In vitro fertilization in Norfolk, Virginia, 1980-1983. J In Vitro Fert Embryo Transf. (1984) 1:24-8. doi: 10.1007/BF01129616

4. Surrey ES, Schoolcraft WB. Evaluating strategies for improving ovarian response of the poor responder undergoing assisted reproductive techniques. Fertil Steril. (2000) 73:667-76. doi: 10.1016/S0015-0282(99)00630-5

5. Polyzos NP, Devroey P. A systematic review of randomized trials for the treatment of poor ovarian responders: is there any light at the end of the tunnel? Fertil Steril. (2011) 96:1058-61. doi: 10.1016/j.fertnstert.2011.09.048

6. Sunkara SK, Tuthill J, Khairy M, El-Toukhy T, Coomarasamy A, Khalaf Y, et al. Pituitary suppression regimens in poor responders undergoing IVF treatment: a systematic review and meta-analysis. Reprod Biomed Online. (2007). 15:539-46. doi: 10.1016/S1472-6483(10)60386-0

7. Ferraretti AP, La Marca A, Fauser BC, Tarlatzis B, Nargund G, Gianaroli $\mathrm{L}$ et al. ESHRE consensus on the definition of 'poor response' to ovarian stimulation for in vitro fertilization: the Bologna criteria. Hum Reprod. (2011). 26:1616-24. doi: 10.1093/humrep/der092

8. POSEIDON Group (Patient-Oriented Strategies Encompassing IndividualizeD Oocyte Number), Alviggi C, Andersen CY, Buehler K, Conforti A, De Placido G, et al. A new more detailed stratification of low responders to ovarian stimulation: from a poor ovarian response to a low prognosis concept. Fertil Steril. (2016) 105:1452-3. doi: 10.1016/j.fertnstert.2016.02.005

9. Papathanasiou A. Implementing the ESHRE 'poor responder' criteria in research studies: methodological implications. Hum Reprod. (2014). 29:18358. doi: 10.1093/humrep/deu135

10. La Marca A, Grisendi V, Giulini S, Sighinolfi G, Tirelli A, Argento C, et al. Live birth rates in the different combinations of the Bologna criteria poor ovarian responders: a validation study. J Assist Reprod Genet. (2015) 32:9317. doi: 10.1007/s10815-015-0476-4

11. Busnelli A, Papaleo E, Del Prato D, La Vecchia I, Iachini E, Paffoni A, et al. A retrospective evaluation of prognosis and cost-effectiveness of IVF in poor responders according to the Bologna criteria. Hum Reprod. (2015). 30:315-22. doi: 10.1093/humrep/deu319

12. Humaidan P, Chin W, Rogoff D, D'Hooghe T, Longobardi S, Hubbard J, et al. Efficacy and safety of follitropin alfa/lutropin alfa in ART: a randomized controlled trial in poor ovarian responders. Hum Reprod. (2017). 32:54455. doi: 10.1093/humrep/dew360

13. Drakopoulos P, Vuong TNL, Ho NAV, Vaiarelli A, Ho MT, Blockeel C, et al. Corifollitropin alfa followed by highly purified HMG versus recombinant FSH in young poor ovarian responders: a multicentre randomized controlled clinical trial. Hum Reprod. (2017) 32:2225-33. doi: 10.1093/humrep/d ex296

14. La Marca A, Sunkara SK. Individualization of controlled ovarian stimulation in IVF using ovarian reserve markers: from theory to practice. Hum Reprod Update. (2014) 20:124-40. doi: 10.1093/humupd/dmt037

15. Broekmans FJ, Kwee J, Hendriks DJ, Mol BW, Lambalk CB. A systematic review of tests predicting ovarian reserve and IVF outcome. Hum Reprod Update. (2006). 12:685-718. doi: 10.1093/humupd/dml034

16. Broer SL, van Disseldorp J, Broeze KA, Dolleman M, Opmeer BC, Bossuyt $\mathrm{P}$, et al. Added value of ovarian reserve testing on patient characteristics in the prediction of ovarian response and ongoing pregnancy: an individual patient data approach. Hum Reprod Update. (2013) 19:2636. doi: 10.1093/humupd/dms041

17. Broer S, Madeleine D, Disseldorp J, Broeze KA, Opmeer BC, Patrick $\mathrm{MM}$, et al. Prediction of an excessive response in in vitro fertilization from patient characteristics and ovarian reserve tests and comparison in subgroups: an individual patient data meta-analysis. Fertil Steril. (2013) 100:420-9. doi: 10.1016/j.fertnstert.2013.04.024
18. Polyzos NP, Sunkara SK. Sub-optimal responders following controlled ovarian stimulation: an overlooked group? Hum Reprod. (2015) 30:20058. doi: 10.1093/humrep/dev149

19. Conforti A, Esteves SC, Cimadomo D, Vaiarelli A, Di Rella F, Ubaldi FM, et al. Management of women with an unexpected low ovarian response to gonadotropin. Front Endocrinol. (2019) 10:387. doi: 10.3389/fendo.2019.00387

20. van Loendersloot LL, van Wely M, Limpens J, Bossuyt PM, Repping S, van der Veen F. Predictive factors in in vitro fertilization (IVF):a systematic review and meta-analysis. Hum Reprod Update. (2010) 16:57789. doi: 10.1093/humupd/dmq015

21. Sunkara SK, Rittenberg V, Raine-Fenning N, Bhattacharya S, Zamora J, Coomarasamy A. Association between the number of eggs and live birth in IVF treatment: an analysis of 400135 treatment cycles. Hum Reprod. (2011) 26:1768-74. doi: 10.1093/humrep/der106

22. Steward RG, Lan L, Shah AA, Yeh JS, Price TM, Goldfarb JM, et al. Oocyte number as a predictor for ovarian hyperstimulation syndrome and live birth: an analysis of 256,381 in vitro fertilization cycles. Fertil Steril. (2014) 101:96773. doi: 10.1016/j.fertnstert.2013.12.026

23. Magnusson Å, Källen K, Thurin-Kjellberg A, Bergh C. The number of oocytes retrieved during IVF: a balance between efficacy and safety. Hum Reprod. (2018). 33:58-64. doi: 10.1093/humrep/dex334

24. Polyzos NP, Drakopoulos P, Parra J, Pellicer A, Santos-Ribeiro S, Tournaye H, et al. Cumulative live birth rates according to the number of oocytes retrieved after the first ovarian stimulation for in vitro fertilization/intracytoplasmic sperm injection: a multicenter multinational analysis including $\sim 15,000$ women. Fertil Steril. (2018). 110:661-70. doi: 10.1016/j.fertnstert.2018.04.039

25. Ata B, Kaplan B, Danzer H, Glassner M, Opsahl M, Tan SL, et al. Array CGH analysis shows that aneuploidy is not related to the number of embryos generated. Reprod Biomed Online. (2012) 24:61420. doi: 10.1016/j.rbmo.2012.02.009

26. Esteves SC, Alviggi C, Humaidan P, Fischer R, Andersen CY, Conforti A, et al. The POSEIDON criteria and its measure of success through the eyes of clinicians and embryologists. Front Endocrinol. (2019). 10:814. doi: 10.3389/fendo.2019.00814

27. Perez Mayorga M, Gromoll J, Behre HM, Gassner C, Nieschlag E, Simoni M. Ovarian response to follicle-stimulating hormone (FSH) stimulation depends on the FSH receptor genotype. J Clin Endocrinol Metab. (2000). 85:33659. doi: $10.1210 /$ jc. 85.9 .3365

28. Gallot V, Berwanger da Silva AL, Genro V, Grynberg M, Frydman N, Fanchin R. Antral follicle responsiveness to follicle-stimulating hormone administration assessed by the Follicular Output RaTe (FORT) may predict in vitro fertilization-embryo transfer outcome. Hum Reprod. (2012). 27:106672. doi: 10.1093/humrep/der479

29. Lehert P, Schertz JC, Ezcurra D. Recombinant human follicle-stimulating hormone produces more oocytes with a lower total dose per cycle in assisted reproductive technologies compared with highly purified human menopausal gonadotrophin: a meta-analysis. Reprod Biol Endocrinol. (2010) 8:112. doi: 10.1186/1477-7827-8-112

30. Alviggi C, Pettersson K, Longobardi S, Andersen CY, Conforti A, De Rosa P, et al. A common polymorphic allele of the LH beta-subunit gene is associated with higher exogenous FSH consumption during controlled ovarian stimulation for assisted reproductive technology. Reprod Biol Endocrinol. (2013) 11:51. doi: 10.1186/1477-7827-11-51

31. Oudendijk JF, Yarde F, Eijkemans MJ, Broekmans FJ, Broer SL. The poor responder in IVF: is the prognosis always poor? A systematic review. Hum Reprod Update. (2012) 18:1-11. doi: 10.1093/humupd/dmr037

32. Siristatidis CS, Gibreel A, Basios G, Maheshwari A, Bhattacharya S. Gonadotrophin-releasing hormone agonist protocols for pituitary suppression in assisted reproduction. Cochrane Database Syst Rev. (2015) 2015:CD006919. doi: 10.1002/14651858.CD006919.pub4

33. Lambalk CB, Banga FR, Huirne JA, Toftager M, Pinborg A, Homburg R, et al. GnRH antagonist versus long agonist protocols in IVF: a systematic review and meta-analysis accounting for patient type. Hum Reprod Update. (2017) 23:560-79. doi: 10.1093/humupd/dmx017

34. Lunenfeld B, Bilger W, Longobardi S, Kirsten J, D'Hooghe T, Sunkara SK. Decision points for individualized hormonal stimulation with recombinant 
gonadotropins for treatment of women with infertility. Gynecol Endocrinol. (2019). 35:1027-36. doi: 10.1080/09513590.2019.1650345

35. Alviggi C, Conforti A, Esteves SC, Andersen CY, Bosch E, Bühler $\mathrm{K}$, et al. Recombinant luteinizing hormone supplementation in assisted reproductive technology: a systematic review. Fertil Steril. (2018). 109:64464. doi: 10.1016/j.fertnstert.2018.01.003

36. Alviggi C, Conforti A, Santi D, Esteves SC, Andersen CY, Humaidan P, et al. Clinical relevance of genetic variants of gonadotropins and their receptors in controlled ovarian stimulation: a systematic review and metaanalysis. Hum Reprod Update. (2018) 24:599-614. doi: 10.1093/humupd/d my019

37. Vaiarelli A, Cimadomo D, Trabucco E, Vallefuoco R, Buffo L, Dusi L, et al. Double stimulation in the same ovarian cycle (DuoStim) to maximize the number of oocytes retrieved from poor prognosis patients: a multicenter experience and SWOT analysis. Front Endocrinol. (2018) 9:317. doi: $10.3389 /$ fendo.2018.00317

38. Vaiarelli A, Cimadomo D, Argento C, Ubaldi N, Trabucco E, Drakopoulos P, et al. Double stimulation in the same ovarian cycle (DuoStim) is an intriguing strategy to improve oocyte yield and the number of competent embryos in a short timeframe. Minerva Ginecol. (2019). 71:372-6. doi: 10.23736/S0026-4784.19. 04390-9

39. Baerwald AR, Adams GP, Pierson RA. Ovarian antral folliculogenesis during the human menstrual cycle: a review. Hum Reprod Update. (2012) 18:7391. doi: 10.1093/humupd/dmr039
40. Ubaldi FM, Capalbo A, Vaiarelli A, Cimadomo D, Colamaria S, Alviggi C, et al. Follicular versus luteal phase ovarian stimulation during the same menstrual cycle (DuoStim) in a reduced ovarian reserve population results in a similar euploid blastocyst formation rate: new insight in ovarian reserve exploitation. Fertil Steril. (2016) 105:1488-95. doi: 10.1016/j.fertnstert.2016.03.002

41. Cimadomo D, Vaiarelli A, Colamaria S, Trabucco E, Alviggi C, Venturella R, et al. Luteal phase anovulatory follicles result in the production of competent oocytes: intra-patient paired case-control study comparing follicular versus luteal phase stimulations in the same ovarian cycle. Hum Reprod. (2018) 33:1442-8. doi: 10.1093/humrep/dey217

42. Boots CE, Meister M, Cooper AR, Hardi A, Jungheim ES. Ovarian stimulation in the luteal phase: systematic review and meta-analysis. J Assist Reprod Genet. (2016). 33:971-80. doi: 10.1007/s10815-016-0721-5

Conflict of Interest: The authors declare that the research was conducted in the absence of any commercial or financial relationships that could be construed as a potential conflict of interest.

Copyright $\odot 2020$ Sunkara, Ramaraju and Kamath. This is an open-access article distributed under the terms of the Creative Commons Attribution License (CC BY). The use, distribution or reproduction in other forums is permitted, provided the original author(s) and the copyright owner(s) are credited and that the original publication in this journal is cited, in accordance with accepted academic practice. No use, distribution or reproduction is permitted which does not comply with these terms. 